Published in final edited form as:

Eur J Clin Nutr. 2019 November 01; 73(11): 1492-1500. doi:10.1038/s41430-019-0393-1.

\title{
Plasma vitamin $\mathbf{C}$ concentrations and risk of incident respiratory diseases and mortality in the European Prospective Investigation into Cancer-Norfolk population-based cohort study
}

\author{
Professor Phyo Kyaw Myint [Professor of Medicine of Old Age] \\ School of Medicine, Medical Sciences \& Nutrition, University of Aberdeen, Aberdeen, UK; \\ Norwich Medical School, University of East Anglia, Norwich Research Park, Norwich, UK; Clinical \\ Gerontology Unit, Department of Public Health and Primary Care, School of Clinical Medicine, \\ University of Cambridge, Cambridge, UK
}

Professor Andrew M Wilson [Professor of Respiratory Medicine], Dr Allan B Clark [Senior Lecturer in Medical Statistics]

Norwich Medical School, University of East Anglia, Norwich Research Park, Norwich, UK

Andrew M Wilson: a.m.wilson@uea.ac.uk; Allan B Clark: Allan.clark@uea.ac.uk

\author{
Mr Robert N Luben [Senior Research Associate], \\ Clinical Gerontology Unit, Department of Public Health and Primary Care, School of Clinical \\ Medicine, University of Cambridge, Cambridge, UK \\ Professor Nicholas J Wareham [Director of MRC Epidemiology Unit], \\ MRC Epidemiology Unit, Cambridge, UK \\ Professor Kay-Tee Khaw [Professor of Clinical Gerontology] \\ Clinical Gerontology Unit, Department of Public Health and Primary Care, School of Clinical \\ Medicine, University of Cambridge, Cambridge, UK \\ Robert N Luben: Robert.luben@srl.cam.ac.uk; Nicholas J Wareham: Nick.wareham@mrc-epid.cam.ac.uk; Kay-Tee Khaw: \\ Kk101@medschl.cam.ac.uk
}

\begin{abstract}
Background-Cancerous and non-cancerous respiratory diseases are common and contribute significantly to global disease burden. We aim to quantify the association between plasma vitamin $\mathrm{C}$ concentrations as an indicator of high fruit and vegetable consumption and the risk of incident respiratory diseases and associated mortality in a general population.
\end{abstract}

\footnotetext{
Corresponding author: Phyo Kyaw Myint, Room 4:013Polwarth Building, School of Medicine, Medical Sciences and Nutrition, University of Aberdeen, Foresterhill, Aberdeen, AB25 2ZD, Tel:+44 (0) 1224437957 | Fax: +44 (0) 1224437911 , phyo.myint@abdn.ac.uk.

Contributors

PKM: design, write up, primary responsibility for final content

AMW: design, write up

ABC: data analysis, write up

RNL: data linkage, write up

NJW: design, provide data, write up

KTK: design, provide data, write up

Conflict of interest

None for all authors.
} 
Methods - 19,357 men and women aged 40-79 years without prevalent respiratory diseases at the baseline (1993-1997) and participating in the European Prospective Investigation into Cancer (EPIC)-Norfolk study in the UK were followed through March 2015 for both incidence and mortality from respiratory diseases.

Results-There were a total of 3914 incident events and 407 deaths due to any respiratory diseases (excluding lung cancers), 367 incident lung cancers and 280 lung cancer deaths during the follow up (total person years $>300,000$ years). Cox proportional hazards models showed, persons in the top quartiles of baseline plasma vitamin C concentrations had a $43 \%$ lower risk of lung cancer (HR 0.57; 95\% CI:0.41-0.81) than did those in the bottom quartile, independently of potential confounders. The results are similar for non-cancerous any respiratory disease (HR $0.85 ; 0.77-0.95$ ), chronic respiratory diseases (HR0.81;0.69-0.96), and pneumonia (HR0.70;0.59-0.83). The corresponding values for mortality were $0.54(0.35-0.81)$, $0.81(0.59-1.12), 0.85(0.44-1.66)$, and $0.61(0.37-1.01)$, respectively. Confining analyses to nonsmokers showed $42 \%$ and $53 \%$ risk reduction of non-smoking related lung cancers incidence and death.

Conclusions-Higher levels of vitamin C concentrations as a marker of high fruit and vegetable consumption reduces the risk of cancerous and non-cancerous respiratory illnesses including nonsmoking related cancers incidence and deaths.

\section{Keywords}

Fruit and vegetable consumption; Vitamin C; Lung cancer; Chronic respiratory disease; Pneumonia; Incidence; Mortality

\section{Introduction}

Respiratory diseases are major causes of mortality and morbidity worldwide. In the UK alone, respiratory disease costs the NHS and society $£ 6.6$ billion: $£ 3$ billion in costs to the care system, $£ 1.9$ billion in mortality costs and $£ 1.7$ billion in illness costs [1]. Irreversible progressive chronic respiratory diseases and pneumonia are common in middle and older age and contribute significantly to this burden. Whilst lung cancer incidence is declining in some countries which imposed smoking ban, there remains risk of lung cancers in general as well as the risk of non-smoking related lung cancer.

Whilst the intake of high fruit and vegetables depicted by plasma vitamin $\mathrm{C}$ concentrations are linked to mortality [2] and chronic cardiovascular conditions including stroke [3-7]. Whether such dietary health behaviour is linked to respiratory diseases including pneumonia is less well understood. Plasma vitamin $\mathrm{C}$ levels can be regarded as a reasonably reliable measure of not just the intake of fruit and vegetables, but the in vivo biologically available vitamin $\mathrm{C}$ for human physiological functions because it cannot be synthesized in the body. It is because the main source of vitamin $\mathrm{C}$ is from dietary fruit, vegetables, and plant foods in humans but is easily denatured by food preparation methods (e.g. via cooking in water, roasting, or grilling) [8]. It also has a short half-life $(\approx 30 \mathrm{~min})$ in the blood $[9,10]$. Therefore, random plasma vitamin $\mathrm{C}$ level is most likely to reflect an individual's habitual dietary pattern as well as method of food preparation. 
The relationship between plasma vitamin $\mathrm{C}$ concentrations and the incidence of chronic obstructive pulmonary disease (COPD), pulmonary fibrosis, pneumonia and respiratory mortality including lung cancer deaths, in particular non-smoking related lung cancers and their associated mortality risks were poor understood. We explored these relationships in the European Prospective Investigation into Cancer (EPIC)-Norfolk. Our secondary objective was to examine whether the relationship, if existed, vary by age, sex, smoking status, physical activity level, and lung function assessed by forced expiratory volume in the first second (FEV1). We then further assessed the relationship between higher level of fruit and vegetable consumption and non-smoking related incidence and mortality for these conditions.

\section{Subjects and Methods}

\section{Participants}

Participants were drawn from the EPIC-Norfolk prospective population study. The detailed recruitment method and study protocol of EPIC-Norfolk were described previously [11]. Briefly, all eligible community-dwelling adults (aged 40-79 years at the baseline (1993-1997)) from 35 participating general practices in Norfolk, UK, were invited to participate. A total of 30,445 ( 40\% response) persons provided written consent to participate in the study (99.6\% British Caucasians). The Norwich Local Research Ethics Committee approved the study.

\section{Measurements}

At the time of the baseline survey, participants completed a detailed health and lifestyle questionnaire and attended health check clinic where trained nurses performed clinical assessments. The data collection methods for variables included in this study are described details in the online supplement [12-17].

\section{Assessment of Vitamin C levels}

After overnight storage in a dark box at $4-7{ }^{\circ} \mathrm{C}$, non-fasting blood samples were centrifuged at $2100 \times \mathrm{g}$ for $15 \mathrm{~min}$ at $4{ }^{\circ} \mathrm{C}$. Plasma vitamin $\mathrm{C}$ was measured from blood collected one year later when funding was available using fluorometric assay within 1 week of sampling [18]. The mean coefficients of variation (CV) were $33.2 \mu \mathrm{mol} / \mathrm{L}$ at the lower end and 102.3 $\mu \mathrm{mol} / \mathrm{L}$ at the upper end.

\section{Outcome ascertainment}

Incident cases were ascertained by using death certificate data and hospital record linkage based on UK Office of National Statistics using International Classification of Disease (ICD), revisions 9 and 10 and National Health Service hospital information systems through ENCORE ((ENCORE - East Norfolk COmmission REcord). Accuracy of this method has been previously validated [19]. Hospitalisation record was linked up to end of March 2015 and death was monitored until end March 2015. Conditions of interest are any respiratory conditions (ICD codes 460-519; J00-J99, chronic respiratory conditions ICD codes 490-496; J40-47,pneumonia, ICD 10 J12-J18, B012,B052,B953,B960,B961,J100, J110,J851, Lung cancers (C33-C34) and asthma (J45-J46). Whilst incident and morality from asthma was 
included in all non-cancerous respiratory diseases, it was specifically excluded from outcomes as adult/older age onset asthma may have other mechanistic pathways which are non-dietary.

\section{Statistical analysis}

Statistical analysis was performed using Stata Version 14.1/SE (StatCorLP, USA. Texas, USA). All tests are two-sided and, as the study is exploratory, no adjustment for multiple comparisons has been made. Any participants with missing value for any of the variables included in the analyses were excluded in individual analyses. Plasma vitamin $\mathrm{C}$ concentration was split into quartiles, with cut-points 41,54 , and $66 \mu \mathrm{mol} / \mathrm{L}$. The percentage of predicted normal were calculated for FVC and FEV1 (FVC\% predicted and FEV1\% predicted respectively) based on the Steel and Coal formula [20]. The incidence of respiratory conditions and respiratory related mortality are reported descriptive, firstly overall and then stratified by the quartiles of plasma vitamin $\mathrm{C}$ concentrations.

Cox proportional hazards models were constructed to determine the association between plasma vitamin $\mathrm{C}$ concentrations and the subsequent risk of all respiratory conditions, chronic respiratory disease, and pneumonia. The risks of mortality for each of the selected outcomes were also assessed. To estimate the independent contribution of vitamin C concentration we controlled for 1) age and sex; 2) age, sex, and respiratory function assessed using FVC and FEV1; 3) age, sex, respiratory function, and lifestyle factors (smoking, alcohol consumption, physical activity and BMI); 4) age, sex, respiratory function, lifestyle factors, occupational social class and Townsend index of deprivation; 5) as in model 3 with additional adjustment for prevalent diabetes, myocardial infarction (MI) and stroke; 6) as in model 4 with additional adjustment for history of diabetes, MI and stroke; and finally model 7 was constructed as in model 6 after excluding participants who were taking vitamin C containing supplements.

To assess the differences in the relationship between plasma concentration of vitamin $\mathrm{C}$ at the baseline and the subsequent incidence of respiratory diseases including mortality specifically in different age groups (younger and older ( $<65$ years and $>=65$ years)), sex (men and women), smoking status (current smokers and non/ex-smokers), physical activity levels (inactive and active), and respiratory function (good and poor function; people with good function defined as people in the top quartile of FEV1 values) the stratified analyses were repeated for model 6.

Analyses were then repeated for main analyses for final two models (models 6 and 7) confining first to non-current smokers and then to never-smokers.

\section{Results}

A total of 25,639 individuals attended their first health check in EPIC-Norfolk during 1993-1997, of these 1,410 were excluded as they had an existing diagnosis of cancer leaving 24,229 individuals. Of these, 2205 had self-reported respiratory diseases and/or taking bronchodilators or drugs for asthma or COPD at the baseline; this leaves 22,024 individuals. After exclusion of 2,685 individuals who did not have vitamin $\mathrm{C}$ measurements and further 3 
participants with missing follow-up data we included a total of 19,336 men and women in the current study. The mean follow-up was 16.54 years. The total follow-up period for the incidence was 319,937 person-years and for the mortality outcome was 336,473 personyears. There were a total of 3,914 incident cases and 407 deaths during the respective follow up periods.

Table 1 shows the sample characteristics by quartiles of plasma vitamin $\mathrm{C}$ concentrations. The quartile values were $=<41 \mu \mathrm{mol} / \mathrm{L}, 42-54 \mu \mathrm{mol} / \mathrm{L},>54-=<66.0 \mu \mathrm{mol} / \mathrm{L}$, and $>66.0$ $\mu \mathrm{mol} / \mathrm{L}$ for quartiles $1,2,3$ and 4 , respectively. This shows significant trends for all selected variables across the plasma vitamin $\mathrm{C}$ categories. People with the higher levels of plasma vitamin $\mathrm{C}$ levels were younger, had better baseline lung functions, materially less likely to be deprived, lower proportion of men, less likely to be current smokers and consumed less amount of alcohol, had higher level of education and occupational social class, physically more active, and had lower prevalence of chronic co-morbid conditions. The crude rates for all the selected outcomes suggest a direct relationship between higher baseline plasma vitamin $\mathrm{C}$ concentrations and a better outcome (lower event rates).

Table 2 shows the hazards ratios and their corresponding 95\% confidence intervals for development of incident any respiratory diseases including chronic respiratory diseases (e.g. COPD/pulmonary fibrosis etc.) and pneumonia over the follow up period. Compared to the people in the bottom quartiles, the people in the highest levels of baseline plasma ascorbic concentrations had significant $15 \%, 19 \% 30 \%$ and $47 \%$ relative risk reduction of having any respiratory conditions, chronic respiratory diseases, hospitalised pneumonia, and lung cancer respectively in adjusted model (model 6). The corresponding relative risk reduction for mortality from these conditions were $13 \%, 37 \%$ and $34 \%$ and $52 \%$, respectively, although these did not reach to statistical significance (Table 3 ) with the exception of lung cancer, which showed relative risk reduction of $46 \%$. Excluding those participants who took vitamin $\mathrm{C}$ containing supplements did not alter the association (model 7, Tables $2 \& 3$ ).

Figure 1 (a-d) show the stratified analyses by age groups (younger and older ( $<65$ years and $>=65$ years)), sex (men and women), smoking status (current smokers, non/ex-smokers), physical activity (active, inactive), and respiratory function (good function defined as top quartile of FEV1 value, and poor function defined as the lower three quartiles) for all outcomes examined for those in top quartile of vitamin $\mathrm{C}$ concentrations compared to the bottom quartile. The benefit of high levels of fruit and vegetable consumption depicted by random plasma vitamin $\mathrm{C}$ concentrations was associated with younger age but generally all subgroups showed consistent trends towards benefit with regard to all outcomes examined. People with lower FEV1 also appear to be benefited from higher fruit and vegetable consumption compared to people with better FEV1. With reduced statistical power most of the sub-analyses shown in Supplementary Table 1 for mortality outcome, whilst most of the estimates did not show any significant benefit with regard to mortality due to these conditions but the point estimates are in consistent with potential benefit across age group, sex, smoking status, physical activity, and the level of FEV1.

Supplementary Table 2 shows the results confining to non-smokers and never-smokers for both incidence and mortality of all these respiratory diseases. With lower numbers whilst the 
95\% CI are wider, the point estimates show similar direction. Of note, the non-smoking related lung cancer incidence and mortality showed stronger benefit $42 \%$ and $53 \%$ relative risk reduction in non-current smokers in people with top quartiles of vitamin $\mathrm{C}$ concentrations compared to the bottom quartile. Supplementary Figure 1 shows the KaplanMeier estimates of survival based on different events. The graphs are top-left for all respiratory events, top right for lung cancer events, bottom-left for chronic respiratory disease event, and bottom right for pneumonia events.

\section{Discussion}

Plasma vitamin C concentration is a good biomarker of plant food, namely fruit and vegetables intake. We found for the first time that the higher habitual intake of fruit and vegetables depicted by higher plasma vitamin $\mathrm{C}$ concentrations at the baseline predict future incidence of any respiratory diseases (excluding cancer), chronic respiratory diseases and hospitalised pneumonia in a general population. Further we demonstrate significant relative risk reduction in lung cancer incidence as well as lung cancer related mortality by higher level of baseline plasma vitamin $\mathrm{C}$ concentrations. The observed effects are even more pronounced for non-current smokers. The benefit observed with each higher quartiles of plasma vitamin $\mathrm{C}$ is fairly consistent and the most beneficial relative risk reduction was observed in the top quartile and the cut off point for the highest quartile in this study was $>66 \mu \mathrm{mol} / \mathrm{L}$. Given that one serving of fruit and vegetables in our cohort equates to $20-\mu \mathrm{mol}$ or 1-SD increase in plasma vitamin C concentration [2], it can be extrapolated from our results that at the level of 3.5 portions of fruit and vegetable consumption could lead to significant health benefit within a general population.

It is well recognised that fresh rather than cooked or boiled fruit and vegetables are a richer source of vitamin C. Due to their antioxidant property, higher levels of vitamin C are thought to be associated with reduced inflammatory process/insult. However, there appears to be distinct lack of nutritional interventions in respiratory diseases except vitamin supplementation with only a few short term studies evaluating supplementation with vitamins. The link between vitamin $\mathrm{C}$ intake and lung cancer incidence has been previously investigated [21]. We have furthered this knowledge and shown that high consumption of fruit and vegetables also potentially have substantial survival benefit with relative risk reduction of $46 \%$.

One short term study of Vitamin $\mathrm{C}$ and $\mathrm{E}$ showed no change in lung function in $24 \mathrm{COPD}$ patients [22]. In a larger 5-year trial of more than 20,000 patients with coronary disease, other occlusive arterial disease, or diabetes, there was no difference in lung function or COPD related outcomes [23]. A Cochrane review of vitamin C treatment for pneumonia identified 3 prophylactic studies conducted more than 30 years ago involving 2335 people from which 37 developed community-acquired pneumonia. In all of these studies there was a significant reduction in the development of pneumonia however only one study (of 674 marine recruits) was a randomised trial and the evidence was felt to be too weak to advocate prophylactic use of vitamin $\mathrm{C}$ to prevent pneumonia in the general population [24]. 
Data from the Third National Health and Nutrition Examination Survey, a US populationbased cross-sectional study suggest that serum vitamin $\mathrm{C}$ concentration is independently related to lung function as assessed by FEV1 [25] whereas serum vitamin C concentration is associated with FVC in patients with airflow obstruction [26]. Other authors have shown the concentration of vitamin $\mathrm{C}$ to be inversely related to all-cause mortality in adults with obstructive lung disease [27] and mean intake of vitamin $\mathrm{C}$ is significantly lower in patients with COPD than controls [28].

Reactive oxygen species are produced by inflammatory cells including neutrophils and macrophages or are inhaled as environmental agents or cigarette smoke. Oxidants result in cellular damage and may result in apoptosis or cellular necrosis, induce mucous secretion or alter remodelling of extracellular matrix [29] all of which may contribute to the pathophysiology of obstructive lung diseases. Indeed, vitamin $\mathrm{C}$ has recently be shown to prevent smoke-induced emphysema in mice that cannot synthesise vitamin $\mathrm{C}$ suggesting that vitamin $C$ reduced the oxidative stress caused by cigarette smoking [30] and therefore are not simply a reflection of diet. Interestingly, in the current study, people in the lowest quartile of vitamin $\mathrm{C}$ had the highest proportion of those with smoking history - Vitamin C has been shown to be required to have an adequate immune response to influenza virus infection in mice [31] and micronutrient deficiencies (including vitamin C) are associated with impaired immune response and higher burden of respiratory infections in elderly humans [32].

There are some limitations which worth discussing. The initial response rate was modest ( $40 \%)$. Nevertheless, the baseline characteristics of the study population were similar to those of other UK population samples, with the exception of slightly lower prevalence of smokers [11] which may be related to healthy responder bias. Whilst the lower prevalence of smoking may have some impact on vitamin $\mathrm{C}$ levels, the internal relationships between vitamin $\mathrm{C}$ levels and incidence respiratory diseases are unlikely to be affected by this. Moreover, the truncation of distribution is more likely to attenuate the associations. . Although follow-up is virtually complete, using hospital record and death certificate linkage approach may underestimate events that are not admitted to the hospital e.g. milder forms of pneumonia.

The use of self-reported respiratory conditions may have missed some prevalent respiratory conditions. Plasma vitamin $\mathrm{C}$ and other covariates, were made at baseline. These measures as well as lifestyle behaviours, which may affect vitamin $\mathrm{C}$ concentration, may have changed over the follow-up period. Due to missing data in some of the variables included in the models, the number of participants included in the analysis reduced with higher level of adjustments but the findings were consistent and adjustments did not particularly attenuate the results.

We addressed confounding and reverse causality issues by comprehensive adjustment of possible confounders. We also performed sensitivity analysis for supplements usage. The incident cases were identified after 1998, at least one year after the baseline. Prospective relationship between baseline vitamin $\mathrm{C}$ concentrations and incidence and mortality also suggests potential causal link, or at least the marker of the conditions examined in this study. 
We performed sensitivity analyses by confining analyses to those who were non-current smokers and never-smokers.

To conclude, we found that high plasma vitamin $\mathrm{C}$ concentrations at baseline are significantly associated with the lower incidence of lung cancer, any respiratory illness other than lung cancer, chronic respiratory diseases and pneumonia. Additionally, those in highest consumption of fruit and vegetables (Quartile 4 of plasma vitamin C concentrations) also had $46 \%$ relative risk reduction from lung cancer mortality (53\% in those who were noncurrent smokers at the baseline) compared to the bottom quartile. Therefore, the plasma vitamin $\mathrm{C}$ level may be useful in identification of those at risk of these both cancerous and non-cancerous respiratory diseases at the population level. As vitamin $\mathrm{C}$ level in plasma is associated with higher fruit and vegetable consumption, increased consumption of fruit and vegetables may reduce the risk of these respiratory conditions and associated health care cost globally.

\title{
Supplementary Material
}

Refer to Web version on PubMed Central for supplementary material.

\section{Acknowledgements}

The authors would like to thank the participants of the EPIC-Norfolk cohort. We thank the nutritionist team and data management team of the EPIC-Norfolk cohort. The EPIC-Norfolk study was supported by grants from the Medical Research Council and Cancer Research UK. Funders and sponsors had no role in design and the data collection, analysis, and interpretation of data and the writing of the article and the decision to submit it for publication.

Sources of support

The EPIC-Norfolk study was supported by grants from the Medical Research Council and Cancer Research UK.

\author{
Abbreviations \\ COPD \\ CV \\ ENCORE \\ EPIC-Norfolk \\ FEV1 \\ FFQ \\ FVC \\ ICD \\ TDI \\ Chronic Obstructive Pulmonary Disease \\ Coefficient of variation \\ East Norfolk Commission Record \\ European Prospective Investigation into Cancer-Norfolk \\ Forced expiratory volume in first second \\ Food frequency questionnaire \\ Forced vital capacity \\ International classification of disease \\ Townsend deprivation index
}




\section{References}

[1]. Gupta R, Limb E. The Burden of Lung Disease: A statistics report from the British Thoracic Society (2nd edition). 2006 Jun 28.accessed 27 Nov 2014

[2]. Khaw KT, Bingham S, Welch A, Luben R, Wareham N, Oakes S, et al. Relation between plasma ascorbic acid and mortality in men and women in EPIC-Norfolk prospective study: a prospective population study. European Prospective Investigation into Cancer and Nutrition. Lancet. 2001; 357:657-63. [PubMed: 11247548]

[3]. Myint PK, Luben RN, Welch AA, Bingham SA, Wareham NJ, Khaw KT. Plasma vitamin C concentrations predict risk of incident stroke over $10 \mathrm{y}$ in 20649 participants of the European Prospective Investigation into Cancer Norfolk prospective population study. Am J Clin Nutr. 2008; 87:64-9. [PubMed: 18175738]

[4]. Myint PK, Luben RN, Wareham NJ, Bingham SA, Khaw KT. Combined effect of health behaviours and risk of first ever stroke in 20,040 men and women over 11 years' follow-up in Norfolk cohort of European Prospective Investigation of Cancer (EPIC Norfolk): prospective population study. BMJ. 2009; 338:b349. [PubMed: 19228771]

[5]. Ness AR, Powles JW, Khaw KT. Vitamin C and cardiovascular disease: a systematic review. J Cardiovasc Risk. 1996; 3:513-21. [PubMed: 9100087]

[6]. Yokoyama T, Date C, Kokubo Y, Yoshiike N, Matsumura Y, Tanaka H. Serum vitamin C concentration was inversely associated with subsequent 20-year incidence of stroke in a Japanese rural community. The Shibata Study. Stroke. 2000; 31:2287-94. [PubMed: 11022052]

[7]. Voko Z, Hollander M, Hofman A, Koudstaal PJ, Breteler MM. Dietary antioxidants and the risk of ischemic stroke: the Rotterdam Study. Neurology. 2003; 61:1273-5. [PubMed: 14610137]

[8]. Food Standard Agency. [accessed 15 September 2014] EVM review of vitamin C. Eat well, be well. Helping you make healthier choices. Internet: http://www.food.gov.uk/multimedia/pdfs/ evm_c.pdf

[9]. Hickey, S, Roberts, H. Ascorbate: the science of vitamin C. Napa, CA: Lulu Press, Inc; 2004. (ISBN 1-4116-0724-4.)

[10]. Hickey S, Roberts H. Misleading information on the properties of vitamin C. PLoS Med. 2005; 2:e307. [PubMed: 16173838]

[11]. Day N, Oakes S, Luben R, Khaw KT, Bingham S, Welch A, et al. EPIC-Norfolk: study design and characteristics of the cohort. European Prospective Investigation of Cancer. Br J Cancer. 1999; 80(suppl 1):95-103. [PubMed: 10466767]

[12]. Cox, B, Huppert, F, Whichelow, M. The Health and Lifestyle Survey: seven years on. Aldershot, UK: Dartmouth Publishing Company; 1993.

[13]. Wareham NJ, Jakes RW, Rennie KL, Schuit J, Mitchell J, Hennings S, et al. Validity and repeatability of a simple index derived from the short physical activity questionnaire used in the European Prospective Investigation into Cancer and Nutrition (EPIC) study. Public Health Nutr. 2003; 6:407-13. [PubMed: 12795830]

[14]. Elias, P, Halstead, K, Prandy, K. CASOC: computer-assisted standard occupational coding. London, UK: HMSO; 1993.

[15]. Shohaimi S, Luben R, Wareham N, Day N, Bingham S, Welch A, et al. Residential area deprivation predicts smoking habit independently of individual educational level and occupational social class. A cross sectional study in the Norfolk cohort of the European Prospective Investigation into Cancer (EPIC-Norfolk). J Epidemiol Community Health. 2003; 57:270-6. [PubMed: 12646543]

[16]. Townsend, P, Phillimore, P, Beattie, A. Health and Deprivation: Inequality and the North. Routledge; London: 1988.

[17]. Welch AA, Luben R, Khaw KT, Bingham SA. The CAFE computer program for nutritional analysis of the EPIC-Norfolk food frequency questionnaire and identification of extreme nutrient values. J Hum Nutr Dietet. 2005; 18:99-116.

[18]. Vuilleumier J, Keck E. Fluorometric assay of vitamin C in biological materials using a centrifugal analyser with fluorescence attachment. J Micronutr Anal. 1989; 5:25-34. 
[19]. Sinha S, Myint PK, Luben RN, Khaw KT. Accuracy of death certification and hospital record linkage for identification of incident stroke. BMC Med Res Methodol. 2008; 8:74. [PubMed: 19000303]

[20]. Roca J, Burgos F, Sunyer J, Saez M, Chinn S, Antó JM, et al. References values for forced spirometry. Group of the European Community Respiratory Health Survey. Eur Respir J. 1998; 11:1354-62. [PubMed: 9657579]

[21]. Luo J, Shen L, Zheng D. Association between vitamin C intake and lung cancer: a dose-response meta-analysis. Sci Rep. 2014; 4

[22]. Wu TC, Huang YC, Hsu SY, Wang YC, Yeh SL. Vitamin E and vitamin C supplementation in patients with chronic obstructive pulmonary disease. Int J Vitam Nutr Res. 2007; 77:272-9. [PubMed: 18271282]

[23]. Heart Protection Study Collaborative Group. MRC/BHF Heart Protection Study of antioxidant vitamin supplementation in 20,536 high-risk individuals: a randomised placebo-controlled trial. Lancet. 2002; 360:23-33. [PubMed: 12114037]

[24]. Hemilä H, Louhiala P. Vitamin C for preventing and treating pneumonia. Cochrane Database Syst Rev. 2013; 8

[25]. McKeever TM, Lewis SA, Smit HA, Burney P, Cassano PA, Britton J. A multivariate analysis of serum nutrient levels and lung function. Respir Res. 2008; 9:67. [PubMed: 18823528]

[26]. Ochs-Balcom HM, Grant BJ, Muti P, Sempos CT, Freudenheim JL, Browne RW, et al. Antioxidants, oxidative stress, and pulmonary function in individuals diagnosed with asthma or COPD. Eur J Clin Nutr. 2006; 60:991-9. [PubMed: 16482071]

[27]. Ford ES, Li C, Cunningham TJ, Croft JB. Associations between antioxidants and all-cause mortality among US adults with obstructive lung function. Br J Nutr. 2014; 112:1662-73. [PubMed: 25315508]

[28]. Ahmadi A, Haghighat N, Hakimrabet M, Tolide-ie H. Nutritional evaluation in chronic obstructive pulmonary disease patients. Pak J Biol Sci. 2012; 15:501-5. [PubMed: 24187906]

[29]. ben Anes A, Fetoui H, Bchir S, ben Nasr H, Chahdoura H, Chabchoub E, et al. Increased oxidative stress and altered levels of nitric oxide and peroxynitrite in Tunisian patients with chronic obstructive pulmonary disease: correlation with disease severity and airflow obstruction. Biol Trace Elem Res. 2014; 161:20-31. [PubMed: 25074430]

[30]. Koike K, Ishigami A, Sato Y, Hirai T, Yuan Y, Kobayashi E, et al. Vitamin C prevents cigarette smoke-induced pulmonary emphysema in mice and provides pulmonary restoration. Am J Respir Cell Mol Biol. 2014; 50:347-57. [PubMed: 24032444]

[31]. Li W, Maeda N, Beck MA. Vitamin C deficiency increases the lung pathology of influenza virusinfected gulo-/- mice. J Nutr. 2006; 136:2611-6. [PubMed: 16988135]

[32]. Hamer DH, Sempértegui F, Estrella B, Tucker KL, Rodríguez A, Egas J, et al. Micronutrient deficiencies are associated with impaired immune response and higher burden of respiratory infections in elderly Ecuadorians. J Nutr. 2009; 139:113-9. [PubMed: 19056665] 
(a)

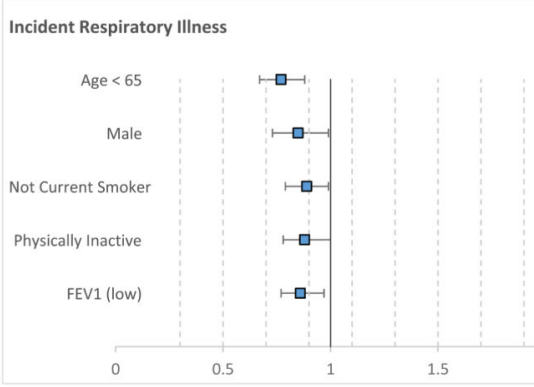

(b)

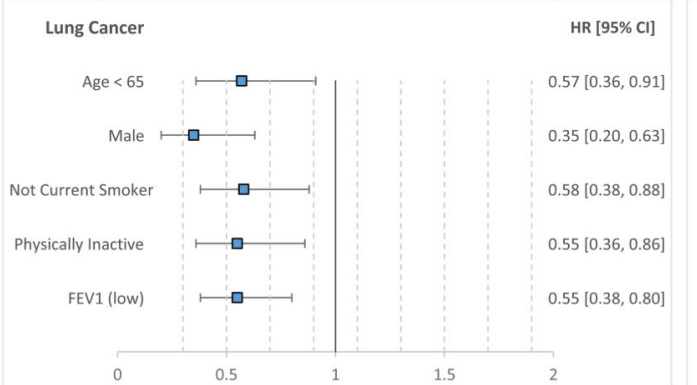

(c)

Chronic Respiratory IIIness

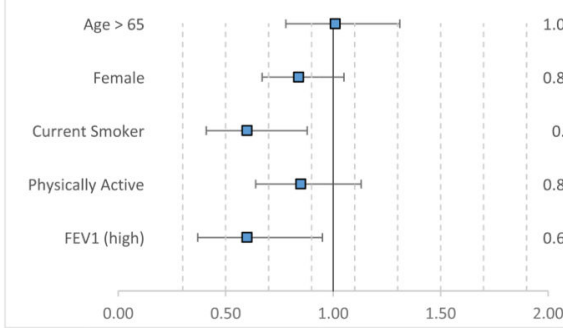

(d)

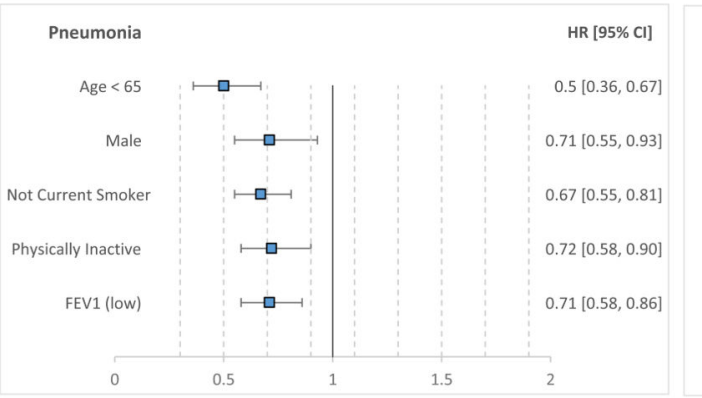

HR [95\% Cl]

$1.01[0.78,1.31]$

$0.84[0.67,1.05]$

$0.6[0.41,0.88]$

$0.85[0.64,1.13]$

$0.60[0.37,0.95]$

00
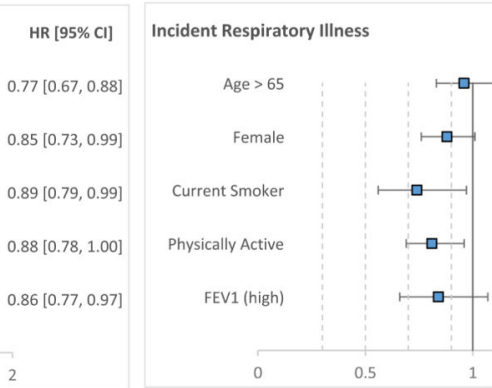

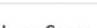

HR $[95 \% \mathrm{Cl}]$

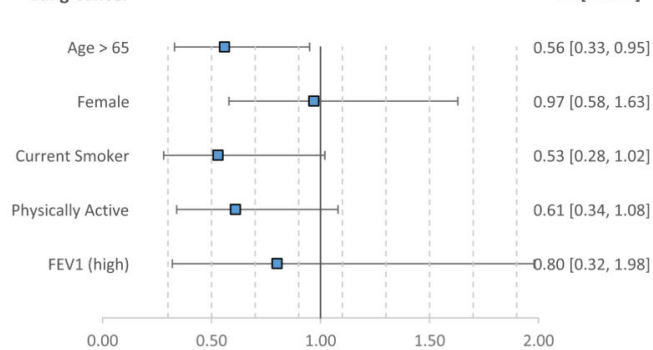

Figure 1. Hazard ratios for study outcomes in people in the highest quartile group (Q4) compared to the lowest quartile group (Q1) stratified by important factors for model 6.

(a): Incident respiratory illness

(b): Incident lung cancer

(c): Incident chronic respiratory diseases

(d): Incident pneumonia

Eur J Clin Nutr. Author manuscript; available in PMC 2020 July 07. 


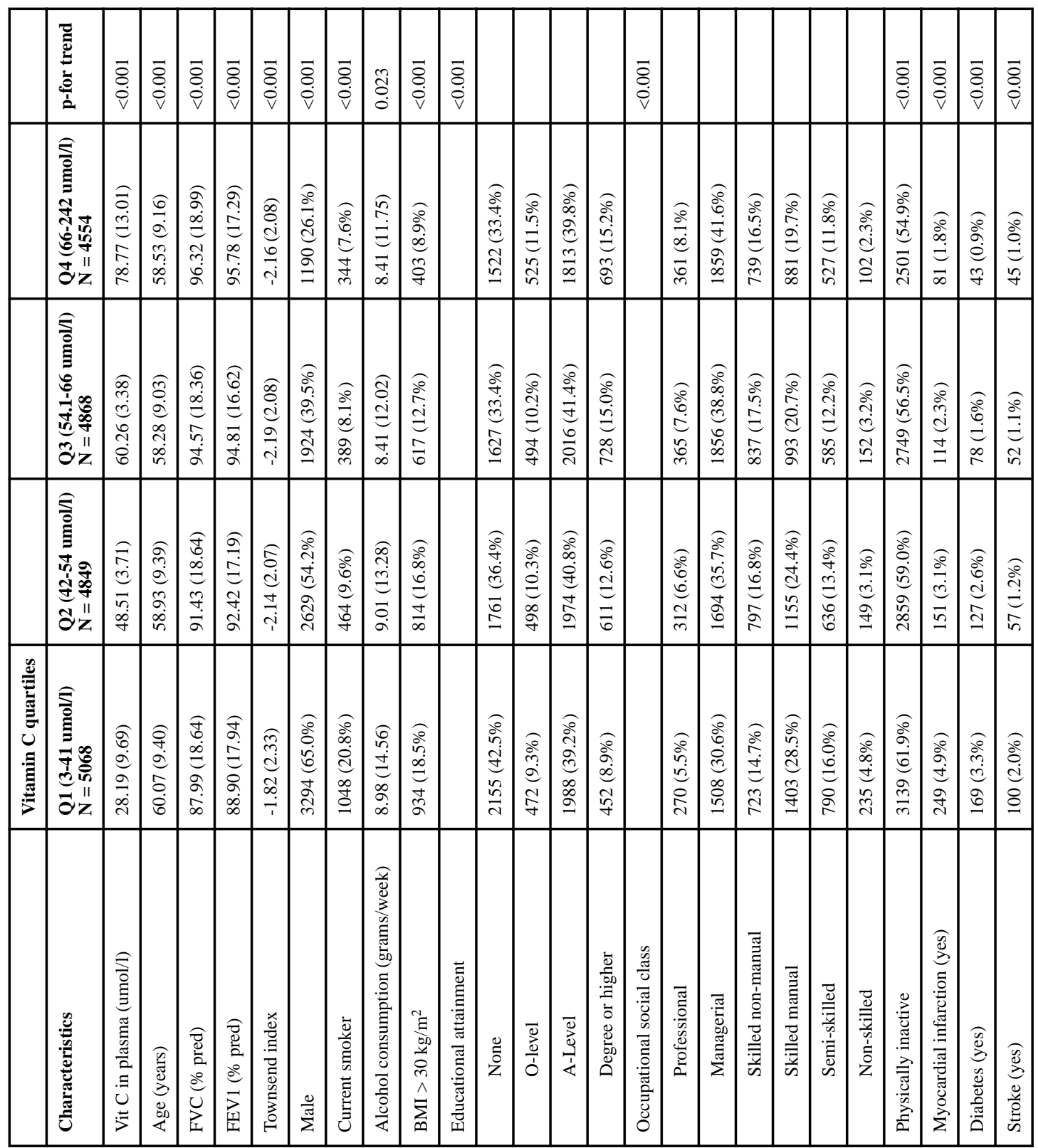




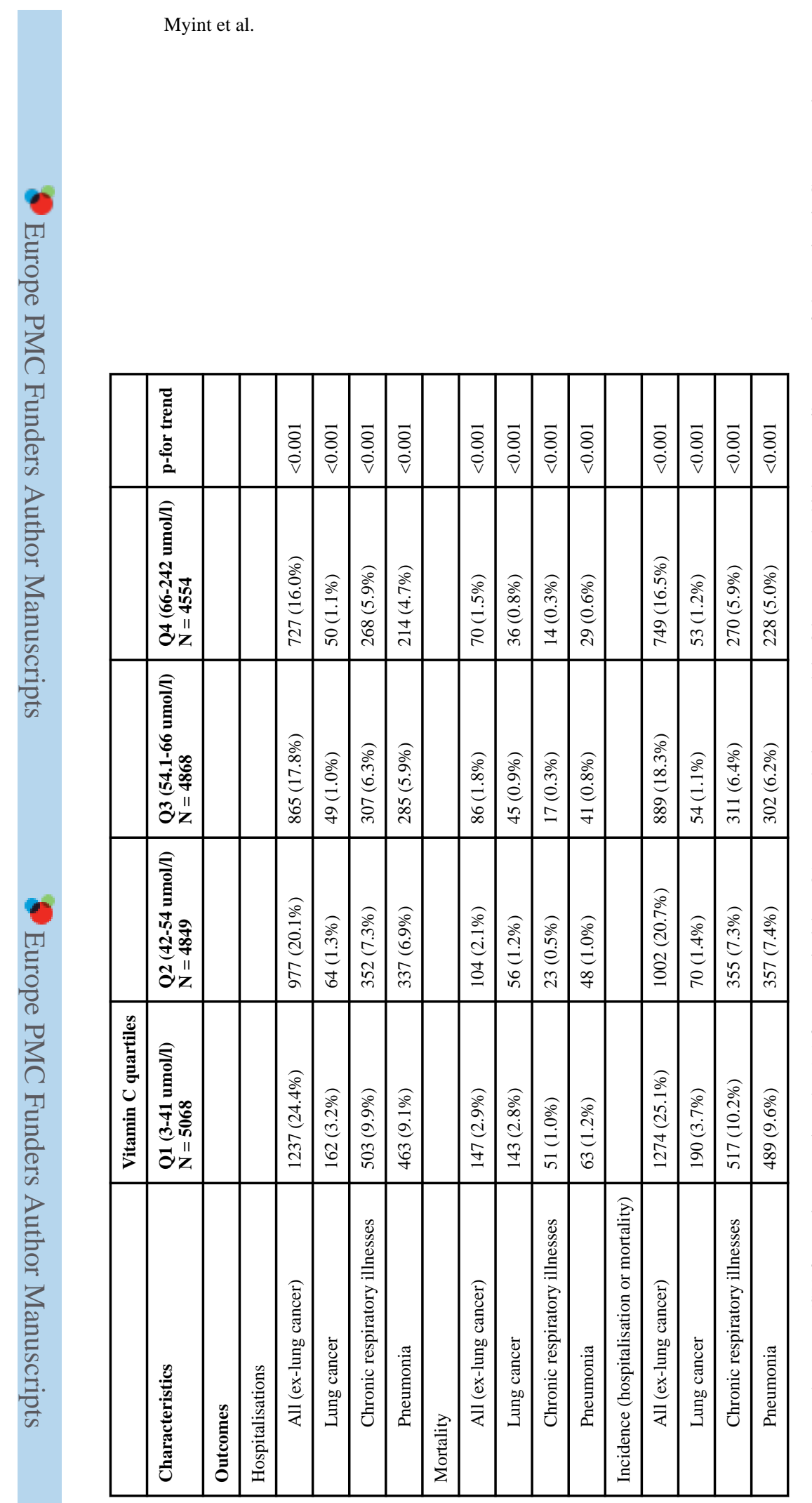

Page 13

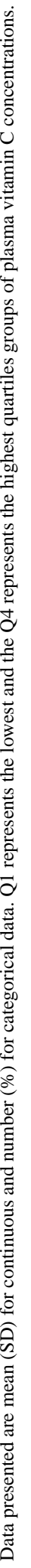




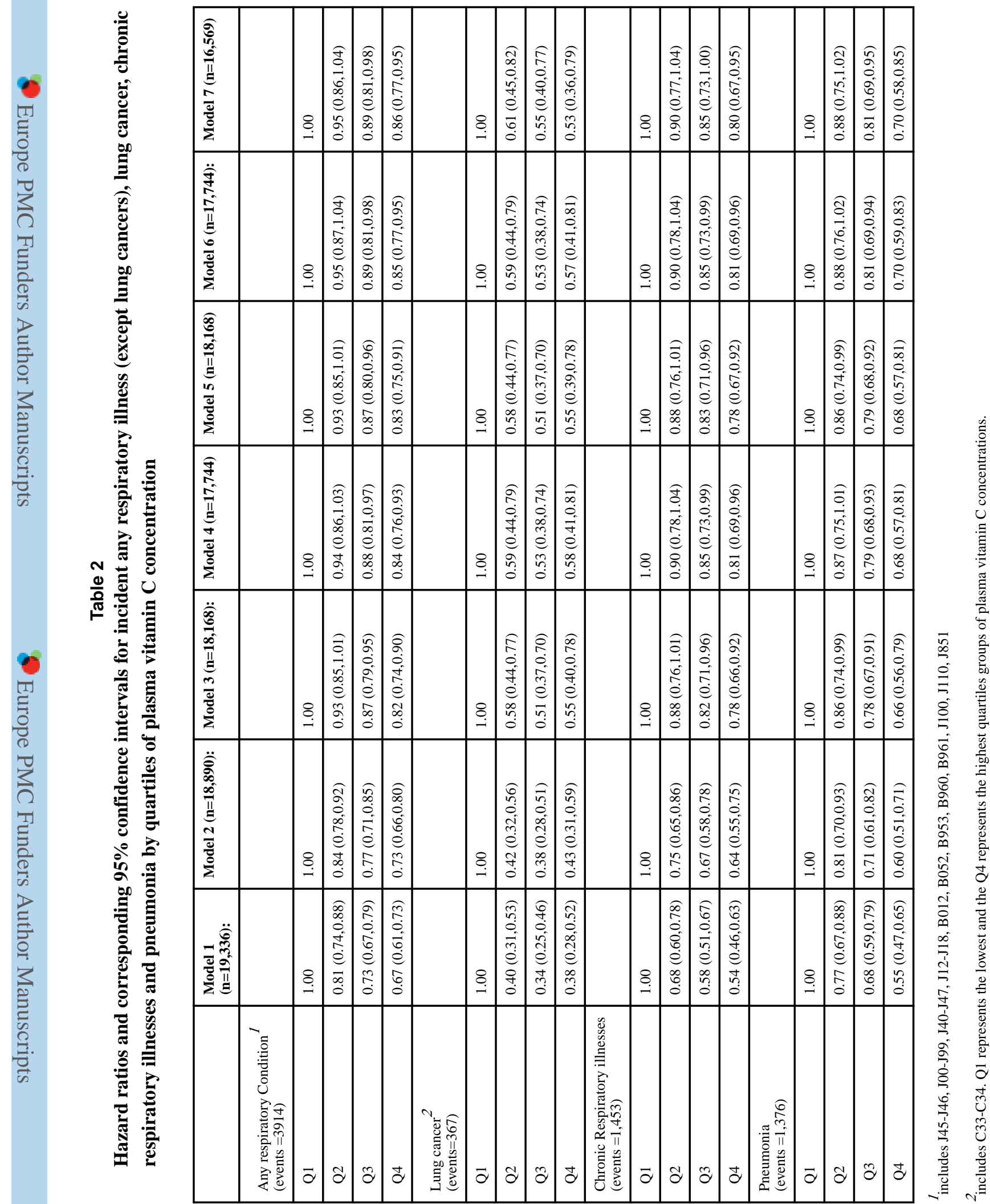




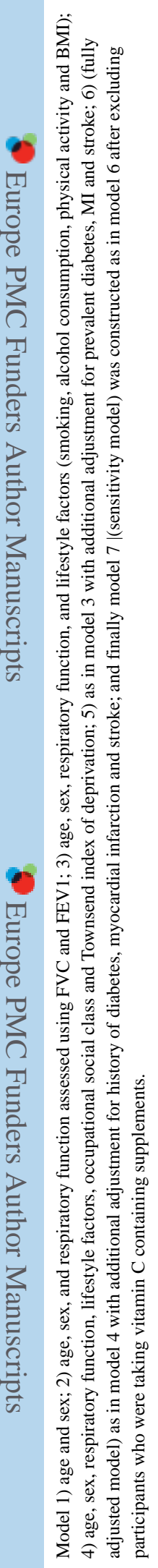

Eur J Clin Nutr. Author manuscript; available in PMC 2020 July 07. 


\begin{tabular}{|c|c|c|c|c|c|c|c|c|c|c|c|c|c|c|c|c|c|c|c|c|c|}
\hline 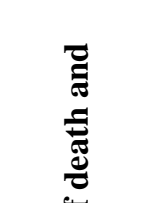 & 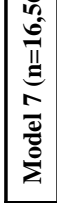 & & $\underset{-}{8}$ & 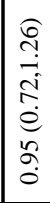 & 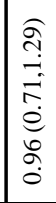 & 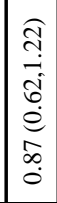 & & $\underset{-}{\stackrel{-}{-}}$ & $\begin{array}{l}\widehat{a} \\
0 \\
0 \\
0 \\
0 \\
0 \\
0 \\
0 \\
0 \\
0\end{array}$ & $\mid \begin{array}{c}\widehat{\alpha} \\
0 \\
0 \\
\hat{j} \\
0 \\
0 \\
0 \\
0 \\
0 \\
0\end{array}$ & \begin{tabular}{|l}
$E$ \\
$E$ \\
0 \\
0 \\
0 \\
0 \\
0 \\
$\infty$ \\
0 \\
0 \\
0
\end{tabular} & & $\stackrel{8}{-}$ & 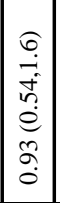 & 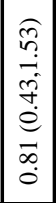 & 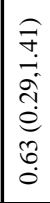 & & $\stackrel{8}{-}$ & 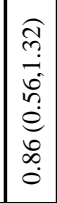 & 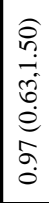 & \begin{tabular}{|c|c|}
0 \\
$\vdots$ \\
0 \\
0 \\
0 \\
0 \\
0 \\
0 \\
0
\end{tabular} \\
\hline 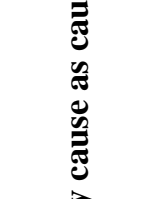 & 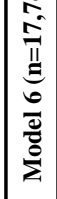 & & $\underset{-}{8}$ & 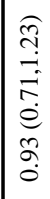 & 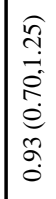 & 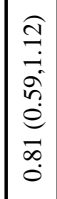 & & $\underset{-}{\stackrel{-}{-}}$ & $\mid \begin{array}{c}\hat{\alpha} \\
0 \\
0 \\
0 \\
0 \\
0 \\
0 \\
0 \\
0 \\
0 \\
0\end{array}$ & $\mid \begin{array}{l}6 \\
0 \\
0 \\
0 \\
+ \\
0 \\
0 \\
0 \\
0 \\
0 \\
0\end{array}$ & 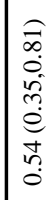 & & $\stackrel{8}{-}$ & 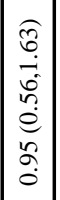 & $\mid \begin{array}{c}0 \\
0 \\
n \\
0 \\
0 \\
0 \\
0 \\
0 \\
0 \\
0 \\
0\end{array}$ & 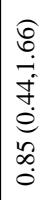 & & $\stackrel{8}{-1}$ & 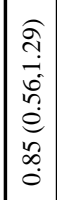 & 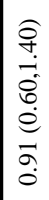 & 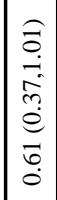 \\
\hline & 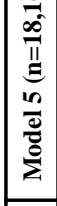 & & $\stackrel{8}{-}$ & \begin{tabular}{|l}
$E$ \\
\\
0 \\
0 \\
0 \\
0 \\
0 \\
0 \\
0 \\
0 \\
\end{tabular} & 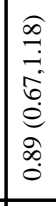 & \begin{tabular}{|l|}
$\hat{a}$ \\
0 \\
0 \\
0 \\
0 \\
$o$ \\
0 \\
0 \\
0 \\
0 \\
0 \\
\end{tabular} & & 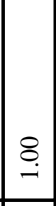 & \begin{tabular}{|l}
$\widehat{\sigma}$ \\
0 \\
0 \\
0 \\
0 \\
0 \\
0 \\
0 \\
0 \\
0 \\
0 \\
\end{tabular} & \begin{tabular}{|c|c}
$\widehat{\infty}$ \\
0 \\
0 \\
+ \\
+ \\
0 \\
$\infty$ \\
0 \\
0 \\
0 \\
\end{tabular} & 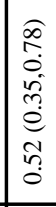 & & $\underset{-}{\stackrel{1}{*}}$ & 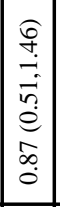 & 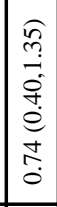 & 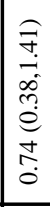 & & $\stackrel{8}{-}$ & 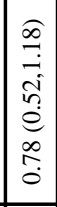 & 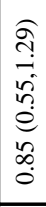 & 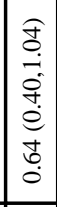 \\
\hline 节 & 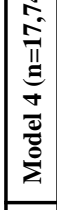 & & $\underset{-}{8}$ & 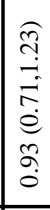 & 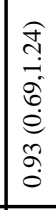 & \begin{tabular}{|c|c}
$\varrho$ \\
$\vdots$ \\
0 \\
0 \\
$e$ \\
0 \\
$\infty$ \\
0 \\
0 \\
0
\end{tabular} & & $\stackrel{8}{-}$ & \begin{tabular}{|c|c}
$\widehat{\alpha}$ \\
0 \\
0 \\
0 \\
0 \\
0 \\
0 \\
0 \\
0 \\
0 \\
0 \\
\end{tabular} & \begin{tabular}{|l|}
0 \\
0 \\
0 \\
0 \\
+7 \\
0 \\
0 \\
0 \\
0 \\
0 \\
0
\end{tabular} & 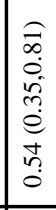 & & $\underset{-}{\stackrel{8}{-}}$ & \begin{tabular}{|c|} 
\\
0 \\
0 \\
0 \\
0 \\
0 \\
0 \\
0 \\
0 \\
0 \\
0 \\
\end{tabular} & 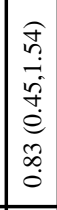 & 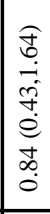 & & $\underset{-}{8}$ & 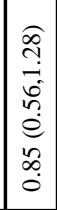 & 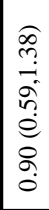 & 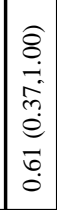 \\
\hline 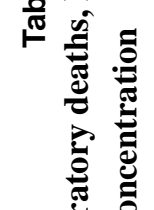 & 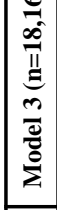 & & $\underset{-}{8}$ & \begin{tabular}{|c}
$E$ \\
\\
0 \\
0 \\
0 \\
0 \\
0 \\
0 \\
0 \\
0
\end{tabular} & 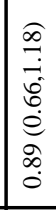 & 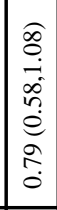 & & $\underset{-}{8}$ & $\begin{array}{l}0 \\
0 \\
\infty \\
0 \\
0 \\
0 \\
0 \\
0 \\
0 \\
0 \\
0 \\
0\end{array}$ & \begin{tabular}{|c|}
$\widehat{\infty}$ \\
0 \\
0 \\
0 \\
0 \\
0 \\
$\infty$ \\
$\infty$ \\
0 \\
0 \\
0
\end{tabular} & 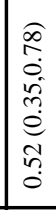 & & $\underset{-}{\stackrel{8}{*}}$ & 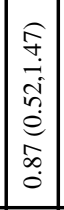 & 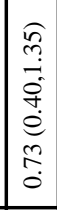 & 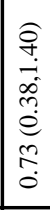 & & $\underset{-}{\varnothing}$ & 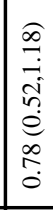 & 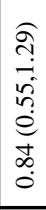 & \begin{tabular}{|c|}
$\widehat{\sigma}$ \\
0 \\
0 \\
0 \\
0 \\
0 \\
+ \\
0 \\
0 \\
0 \\
\end{tabular} \\
\hline 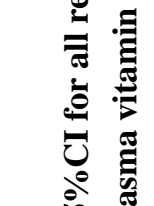 & 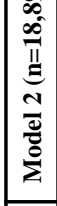 & & $\underset{-}{\stackrel{8}{-}}$ & \begin{tabular}{|l}
$\widehat{0}$ \\
0 \\
0 \\
0 \\
0 \\
0 \\
0 \\
0 \\
0 \\
\end{tabular} & \begin{tabular}{|l}
$\mid$ \\
0 \\
0 \\
0 \\
0 \\
0 \\
0 \\
0 \\
0 \\
0 \\
0 \\
\end{tabular} & \begin{tabular}{|c|}
$\widehat{\sigma}$ \\
0 \\
0 \\
0 \\
0 \\
0 \\
0 \\
0 \\
0 \\
0 \\
\end{tabular} & & $\underset{-}{\stackrel{8}{-}}$ & 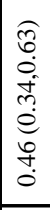 & 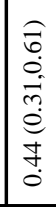 & 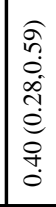 & & $\stackrel{8}{-}$ & 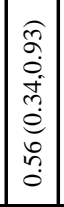 & 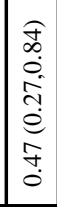 & 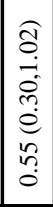 & & $\underset{-}{\stackrel{1}{*}}$ & 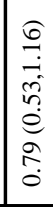 & 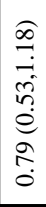 & 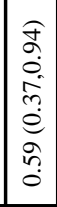 \\
\hline 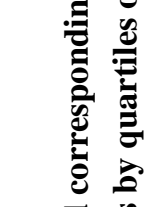 & 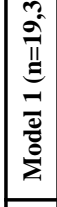 & & $\underset{-}{\stackrel{8}{-}}$ & $\begin{array}{l}\widehat{\sigma} \\
\text { ô } \\
0 \\
0 \\
0 \\
\hat{\theta} \\
\hat{2} \\
0 \\
\end{array}$ & \begin{tabular}{|c|}
$\hat{\sigma}$ \\
0 \\
0 \\
$\hat{n}$ \\
0 \\
0 \\
0 \\
0 \\
0
\end{tabular} & 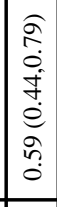 & & $\underset{-}{\stackrel{8}{-}}$ & 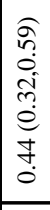 & 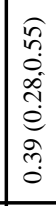 & 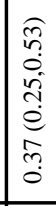 & & $\underset{-}{\stackrel{1}{*}}$ & \begin{tabular}{|c|}
$\widehat{c}$ \\
0 \\
0 \\
0 \\
0 \\
0 \\
0 \\
0 \\
0 \\
0 \\
0 \\
\end{tabular} & 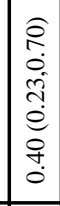 & \begin{tabular}{|c|}
$\widehat{\sigma}$ \\
0 \\
0 \\
0 \\
0 \\
0 \\
0 \\
0 \\
0 \\
\end{tabular} & & $\underset{-}{8}$ & 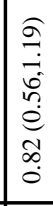 & 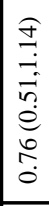 & \begin{tabular}{|c|}
0 \\
0 \\
0 \\
0 \\
0 \\
0 \\
0 \\
0 \\
+ \\
0 \\
0 \\
0 \\
\end{tabular} \\
\hline 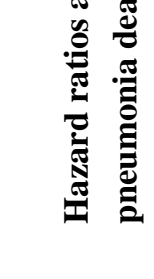 & & 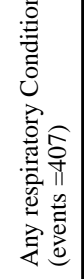 & $\bar{\alpha}$ & $\mathcal{I}$ & $\approx$ & d & 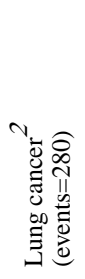 & $\vec{\alpha}$ & a & $\tilde{\partial}$ & $\Xi$ & 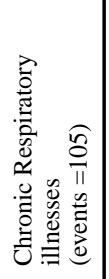 & $\bar{\alpha}$ & $\approx$ & 2 & $\Xi$ & 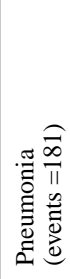 & $\bar{\sigma}$ & 8 & 2 & む \\
\hline
\end{tabular}




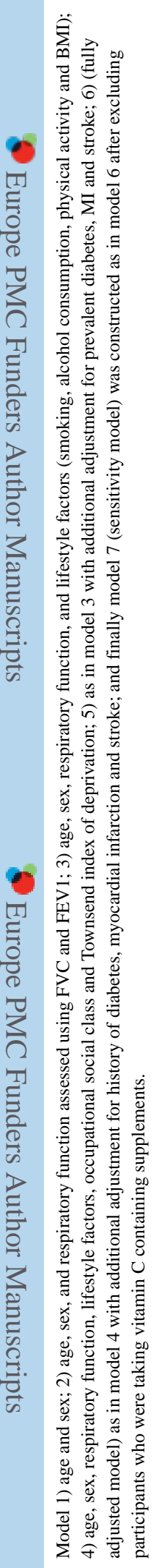

Eur J Clin Nutr. Author manuscript; available in PMC 2020 July 07. 